\title{
Modeling the deployment of plug-in hybrid and electric vehicles and their effects on the Australian National Electricity Market.
}

\author{
Liam Wagner, Member, IEEE \\ The University of Queensland, \\ School of Economics, \\ St Lucia, Brisbane, Qld, \\ Australia 4072 \\ Email: 1.wagner@uq.edu.au
}

\author{
Luke Reedman \\ Carbon Futures \\ CSIRO Energy Transformed Flagship \\ PO BOX 330, Newcastle \\ NSW 2300, Australia \\ Email: Luke.Reedman@csiro.au
}

\begin{abstract}
The development of hybrid and fully electric vehicles could deliver significant reductions of emissions from the Australian transportation sector by shifting its major energy source from internal combustion to electricity. This shift towards the the use of electricity shifts the point source emissions to one which has a lower emissions intensity. Changes in load behaviour as a result of the consumer uptake of these vehicles will have significant consequences for network and central planners for the future of Australia's electricity supply industry. This paper investigates the effects on the security of supply of energy during these previously unseen demand patterns, while also examining changes to spot market prices and changes in emissions rates. The simulation results indicate that wholesale prices during the off-peak period will increase slowly over time with controlled charging. While uncontrolled charging increases the incidence of extreme price events and a considerable number of hours with un-served energy within the network. This increase in spot prices will require further review by policy makers of regulated retail electricity tariffs. We also discuss the implementation of possible changes to the retail tariff structure to accommodate the charging of these vehicles.
\end{abstract}

\section{INTRODUCTION}

Current community concern over climate change and energy security has placed a greater emphasis on finding alternatives to consuming petroleum based products particularly for transportation. Energy production and consumption trends are being reviewed by communities requiring more cost effective and environmentally friendly technologies, particularly for transport. Some automakers have responded announcing production runs of plug-in hybrid $(\mathrm{PH})$ and fully electric vehicles (EVs) to satisfy increasing consumer demands for greater fuel efficiency. It is currently expected that these alternative vehicles will be available for wider adoption in Australia after 2015. Several studies into the benefits and costs of the broad scale deployment of PH and EV's have show the benefits for the shift of point source emissions from oil based fuels to electricity generation [9], [15], [10]. Australia however has yet to be subject to a comprehensive analysis of the benefits, grid impacts and potential costs of the deployment and integration of $\mathrm{PH}$ and EV's into the future.
The focus of this study is to describe via this initial work, is the presentation of energy market modelling frameworks which are best suited to a diverse and very sparse electricity network which encounters different operating and policy conditions to those of North America and Europe. Furth Prior grid based scenario analysis has been performed on a least cost unit commitment basis such as [15]. The simulation of market behaviour and the inevitable change in electricity use trends has been performed using an optimal dispatch model of the National Electricity Market (NEM) that simulates operation and dispatch of power generation assets on a half-hourly basis over the medium term. This model evaluates the dispatch of the optimal fuel type mix based on an order of merit determined by bidding behaviour of generators to recover short run and long run marginal costs (SRMC and LRMC). Marginal cost recovery for generating units is the primary driver for bidding behaviour within the NEM, while possible fuel mix changes due to increased demand during off-peak time periods will also change spot market price behaviour and emissions rates. To estimate the customer uptake of electrified vehicles, we employ a structural model of the energy sector via the transport module of CSIROs Energy Sector Model (ESM).

Furthermore for this study of the effects of grid integrated vehicles on the Australian electricity network, we have developed two vehicle charging scenarios. For each of these scenarios we have developed a half-hourly charging profile for a fleet of PH and EV's. This load profile was then added to a base demand forecast via forecasts of future energy use conducted by the AEMO [3]. After describing each of our charging scenarios we provide fleet average demand profiles and show how each scenario affects prices and available supply.

\section{MODELING}

\section{A. Energy Sector Model}

The Energy Sector Model (ESM) is an Australian energy sector model co-developed by the Commonwealth Scientific and Industrial Research Organisation (CSIRO) and the 
Australian Bureau of Agricultural and Resource Economics (ABARE) in 2006. Since that time CSIRO has significantly modified and expanded ESM. ESM is a partial equilibrium (bottom-up) model of the electricity and transport sectors. The model has a robust economic decision making framework around the cost of alternative fuels and vehicles as well as detailed fuel and vehicle technical performance characterization such as fuel efficiencies and emission factors by transport mode, vehicle type, engine type and age. It also has a detailed representation of the electricity generation sector. In this paper we employ the transport module only.

ESM has been applied in scenario analysis of transport energy futures including: alternative emission targets (e.g., CSIRO, 2008 [6], Graham et al., 2008 [12], Reedman and Graham, 2009 [16]), alternative carbon price regimes (e.g., CSIRO and ABARE, 2006 [5]; Garnaut, 2008 [11]) and peak oil scenarios (Graham and Reedman, 2010 [13]). ESM is solved as a linear program where the objective function is to maximize welfare which is the discounted sum of consumer and producer surplus over time. The sum of consumer and producer surplus is calculated as the integral of the demand functions minus the integral of the supply functions which are both disaggregated into many components across the electricity and transport markets. The objective function is maximised subject to constraints that control for the physical limitations of fuel resources, the stock of electricity plant and vehicles, greenhouse gas emissions as prescribed by legislation, and various market and technology specific constraints such as the need to maintain a minimum number of peaking plants to meet rapid changes in the electricity load. The main components of the transport module of ESM include:

- Coverage of all States and the Northern Territory (Australian Capital Territory is modelled as part of NSW)

- Nine road transport modes: small, medium and heavy passenger cars; small, medium and heavy commercial vehicles; rigid trucks; articulated trucks and buses

- Five engine types: internal combustion; hybrid electric/internal combustion; hybrid plug-in electric/internal combustion; fully electric and fuel cell

- Thirteen road transport fuels: petrol; diesel; liquefied petroleum gas (LPG); natural gas (compressed (CNG) or liquefied (LNG)); petrol with 10 per cent ethanol blend; diesel with 20 per cent biodiesel blend; ethanol and biodiesel at high concentrations; biomass to liquids diesel; gas to liquids diesel; coal to liquids diesel with upstream $\mathrm{CO} 2$ capture; hydrogen (from renewable's) and electricity

- All vehicles are assigned a vintage based on when they were first purchased or installed in annual increments

- Time is represented in annual frequency $(2006,2007, . ., 2050)$.

All technologies are assessed on the basis of their relative costs subject to constraints such as the turnover of capital stock, existing or new policies such as subsidies and taxes. The model aims to mirror real world investment decisions by simultaneously taking into account:

- The requirement to earn a reasonable return on investment over the life of a vehicle

- That consumers react to price signals (price elastic demand)

- That the consumption of energy resources by one user affects the price and availability of that resource for other users, and the overall cost of energy and transport services, and

- Current transport market policy settings including vehicle registration requirements, excise arrangements, biofuel mandates and pollutant emission standards.

The model evaluates uptake on the basis of cost competitiveness but at the same time takes into account the key constraints with regard to the operation of transport markets, current excise and mandated fuel mix legislation, GHG emission limits, existing vehicle stock in each State, and lead times in the availability of new vehicles. It does not take into account issues such as community acceptance of technologies but these can be controlled by imposing various scenario assumptions which constrain the solution to user provided limits. For given time paths of the exogenous (or input) variables that define the economic environment, ESM determines the time paths of the endogenous (output) variables. Key output variables include:

- Fuel and engine technology uptake

- Fuel consumption

- Cost of transport services (for example, cents per $\mathrm{km}$ )

- Price of fuels

- GHG and criteria air pollutant emissions

- Demand for transport services.

Some of these outputs can also be defined as fixed inputs depending upon the design of the scenario. The endogenous variables are determined using demand and production relationships, commodity balance definitions and assumptions of competitive markets at each time step for fuels, electricity and transport services, and over time for assets such as vehicles. With respect to asset markets, the assumption is used that market participants know future outcomes of their joint actions over the entire time horizon of the model.

\section{B. Oil Prices}

The modelling for this paper will employ two oil price projections: the IEA reference price from the World Economic Outlook (IEA, 2009 [14]) and the EIA high price from the Annual Energy Outlook 2010: With Projections to 2035 (EIA, 2010 [7]). The IEA 2009 Reference oil price has oil demand growth of $1 \%$ per year on average over the full projection period, from 85.2 million barrels per day (mb/d) in 2007 (and $84.7 \mathrm{mb} / \mathrm{d}$ in 2008) to $88.4 \mathrm{mb} / \mathrm{d}$ in 2015 and $105.2 \mathrm{mb} / \mathrm{d}$ in 2030. The scenario assumes that crude oil continues to supply the bulk of demand with an increasing share met by natural gas liquids and unconventional oil with an absence of major supply and demand imbalance. Oil prices rise from their current level 
of around US\$70/bbl to US\$115/bbl in 2030 (IEA, 2009). Given our projection period out to 2035 , we extrapolate the price series based on the trend between 2015 and 2030. Based on this method, oil prices reach around US $\$ 125 / \mathrm{bbl}$ in 2035. The AEO2010 high oil price case assumes not only a rebound in world oil prices with the return of world economic growth, but also a continued rapid escalation in prices as a result of long-term restrictions on conventional liquids production. The restrictions result from both political decisions and resource characteristics: the major OPEC and non-OPEC producing countries use quotas, fiscal regimes, and varying degrees of nationalisation to further increase revenues from oil production, and the consuming countries turn to domestic production of high-cost unconventional liquids to satisfy demand. As a result, in the high oil price case, world oil prices rise throughout the projection period, to $\$ 210$ per barrel in 2035 [7].

\section{The Future of Energy Production and its use by Trans- portation}

The future composition of installed generation assets on the NEM has been established using the future planning scenarios performed by the AEMO [3] and via the ESM detailed above. The role out of different electricity supply assets which fulfill current arrangements surrounding the Mandatory Renewable Energy Target (MRET) and the retirement of older less efficient power stations, provides for least cost electricity generation on the NEM. The outputs for generation fleet are detailed in Table I:

The deployment of PH and EV's is determined within the ESM to meet the least cost of the transport needs of Australia. The rates of adoption of these vehicles is detailed in Table II.

\section{Electricity Market Modelling}

To investigate the effects that consumer uptake of PH's might have on the demand for electricity on the NEM, we have used PLEXOS electricity modelling software platform, to simulates the NEM's dispatch and bidding behaviour on a half hourly basis over a ten year time frame. PLEXOS is a commercially available optimization theory based electricity market simulation platform which was developed by Energy Exemplar [8]. At its core is the implementation of rigorous operation algorithms and tools such as Linear Programming (LP) and Mixed Integer Programming (MIP). PLEXOS takes advantage of these tools in combination with an extensive input database of regional demand forecasts, inter-regional transmission constraints and generating plant technical data to produce price, generator and demand forecasts by applying the SPD (scheduling, pricing and dispatch) engine used by the AEMO to operate the NEM.

This model will optimally dispatch generating units based on marginal cost recovery and availability at half hour intervals by using forecasts of system loads. The Short Run Marginal Costs (SRMC) and Long Run Marginal Costs (LRMC) for each generating unit is calculated by using data on fuel costs, O\&M, startup costs and weighted average costs of capital (WACC) from ACIL Tasman [1]. The database that we have used also includes transmission, inter-connector flow, emissions and fuel availability constraints. Furthermore, we are also able to examine marginal loss factors, planned and unplanned outages and unit performance.

Network system load profiles for the South-East Queensland region were used to estimate individual demand behaviour. We then applied alternative charging scenarios to gauge the effects on network demand. To estimate the customer uptake of electrified vehicles, we employ a structural model of the transport sector via the transport module of CSIROs Energy Sector Model (ESM).

Initially we run a base case scenario to establish load forecasts and capacity factors which we shape to historical data obtained from AEMOs data server. We then use our model to establish a benchmark for greenhouse gas (GHG) emissions which provides an emissions profile for the NEM. Having established a base case we move on to establishing a variety of new demand scenarios based on customer uptake of PHEVs and EVs in the Australian NEM.

The development of our study begins with a forecast of network and market behvaiour for our 11 year time frame to establish a base case scenario which we will then compare our results. Forecasts of demand within the NEM were obtained via data available from AEMO [3], [2], allowing us to develop a 50 POE scenario. Network system load profiles for all regions of NEM were also used to produce a forecast of regional demand. Furthermore, generating plant behaviour was obtained from ACIL Tasman [1] and AEMO's data server to produce a forecast for available supply across the NEM, with particular attention to new plant development. While forecasted customer growth in the NEM has been identified by AEMO as a network planning issue, the broad scale deployment of PH and EV's have yet to be seriously considered by the market operator.

Individual plant operating parameters have been obtained from [3], [2] to assist in the population of available plant. This extensive data set includes:

- Capacity factors

- Ramp rates

- Emissions profiles

- Fuel costs

- Variable and fixed operating and management costs

- Scheduled outages

- Probability of forced outage rates

- Transmission inter-connector constraints

\section{E. Charging rates}

For this study of the effects of PHEV's on the Australian electricity network we have developed three vehicle charging scenarios. These scenarios where chosen on the basis of being the most likely given current technology for demand management within the NEM. For each of these scenarios we have developed a half hourly charging profile for a fleet of PHEV's. This load profile was then added to a base demand forecast (examined above in section II-D). After describing 
TABLE I

INSTALLED GENERATION CAPACITY By TECHNOLOGY TYPE (MW) FOR THE PLANNING HORIZON OF 2020-2030

\begin{tabular}{|c|c|c|c|c|c|c|c|c|c|c|c|}
\hline & 2020 & 2021 & 2022 & 2023 & 2024 & 2025 & 2026 & 2027 & 2028 & 2029 & 2030 \\
\hline Brown Coal & 7931 & 8228 & 8216 & 8210 & 8396 & 8586 & 8774 & 8976 & 9235 & 9451 & 9665 \\
\hline Black Coal & 23367 & 25212 & 26839 & 28264 & 28993 & 29751 & 30502 & 31269 & 32006 & 32487 & 33129 \\
\hline IGCC Black Coal & 31 & 30 & 29 & 29 & 28 & 27 & 26 & 25 & 24 & 23 & 23 \\
\hline Combined Cycle Gas Turbine & 3622 & 2304 & 1704 & 1473 & 1496 & 1515 & 1558 & 1588 & 1619 & 1651 & 1693 \\
\hline Open Cycle Gas Turbine & 3347 & 2530 & 2698 & 2860 & 2947 & 3055 & 3113 & 3203 & 3382 & 3142 & 3139 \\
\hline Biomass & 229 & 223 & 201 & 186 & 164 & 155 & 83 & 45 & 57 & 0 & 0 \\
\hline Wind & 10881 & 10844 & 10841 & 10826 & 10806 & 10770 & 10663 & 10560 & 10505 & 10337 & 10249 \\
\hline Hydro & 5988 & 5968 & 5968 & 5968 & 5968 & 5968 & 5968 & 5968 & 5968 & 5968 & 5968 \\
\hline Solar Thermal & 1464 & 1455 & 1447 & 1438 & 1430 & 1422 & 1414 & 1406 & 1398 & 2379 & 2992 \\
\hline Geothermal & 465 & 465 & 465 & 465 & 465 & 465 & 465 & 465 & 465 & 465 & 465 \\
\hline
\end{tabular}

TABLE II

Deployment Rates of Electric and Plug-in Hybid Electric Vehicles on the NEM for 2020 to 2030

\begin{tabular}{|c|c|c|c|c|c|c|c|c|c|c|c|}
\hline & 2020 & 2021 & 2022 & 2023 & 2024 & 2025 & 2026 & 2027 & 2028 & 2029 & 2030 \\
\hline Passenger Light Car: EV & 0 & 0 & 0 & 155175 & 328371 & 547664 & 786178 & 1037875 & 1309878 & 1603171 & 1916546 \\
\hline Light Commercial Vehicle: EV & 0 & 0 & 6711 & 26346 & 47400 & 69932 & 93122 & 118547 & 143896 & 168588 & 192379 \\
\hline Rigid Truck: EV & 35855 & 51236 & 70037 & 93871 & 119669 & 122202 & 124746 & 127316 & 129897 & 132415 & 134953 \\
\hline Medium Commercial Vehicle: PHEV & 0 & 0 & 0 & 50000 & 71499 & 71499 & 71499 & 71499 & 71499 & 72062 & 71874 \\
\hline Heavy Commercial Vehicle: PHEV & 0 & 0 & 0 & 0 & 28501 & 62189 & 99857 & 140453 & 177531 & 209475 & 245081 \\
\hline
\end{tabular}

each of our two charging scenarios we provide fleet average demand profiles and show how each scenario affects prices and available supply.

1) Uncontrolled charging: With the uptake of this new technology, the most likely scenario in the early stages of adoption will be one of uncontrolled charging of PHEV's. This scenario assumes that owners will begin charging within two hours of the end of business hours and will stop when the battery is fully charged (i.e. $7 \mathrm{pm}$ till 1am, see Figure 1). Other recent studies such as Parks et.al. [15], have considered this as a business as usual case where there is no control implemented by the network or by vehicle mechanism.

This case could be regarded as a worst case scenario as it could present several difficulties for network planning with respect to normal consumer peak demand requirements. To establish this case we assume that all vehicles charge at a constant rate of $2 k W / h$ in approximately 6 hours. It should also be noted that for the remaining two scenarios we will use the above charging rates.

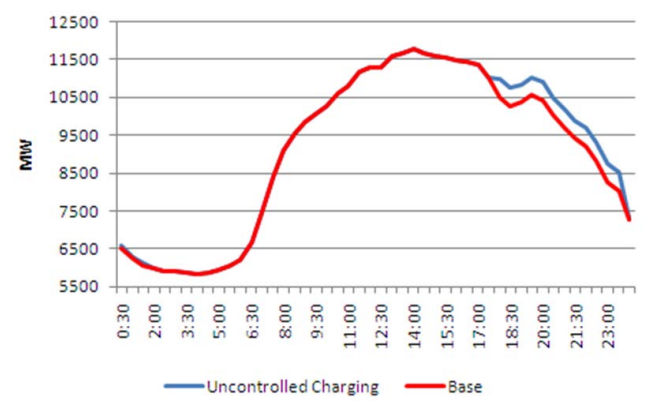

Fig. 1. Typical Demand Profile (RED) with uncontrolled PH and EV charging (Blue)
2) Off-Peak controlled charging: This off-peak scenario will take advantage of a highly controlled (night rate super economy) supply between 12 am to 6am (see Figure 2). This relies on network suppliers being able to control vehicle charging directly or in-directly. Furthermore this case will allow network operation to match charging to periods of minimal demand during off-peak. Similar control of hot water systems has been implemented by network operators Energex and Ergon in Queensland to optimize load control.

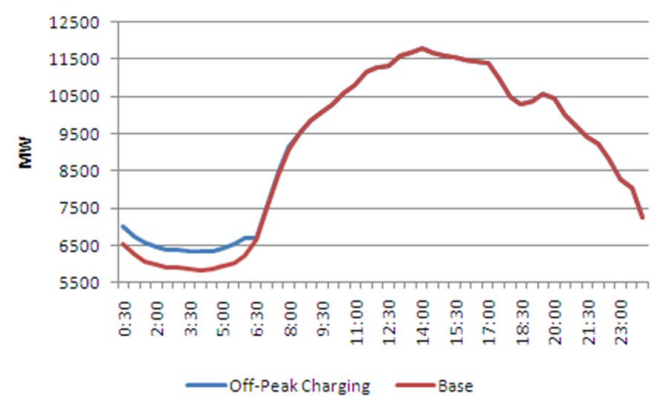

Fig. 2. Typical Demand Profile (RED) with off-peak PH and EV's charging (Blue)

\section{F. Modelling Scenarios}

The evaluation of deployment rates of PH and EV's will performed via four scenarios which take into account the timing of battery charging and oil prices (see Table III). This initial study examines the 11 years of the deployment of different consumer and industrial vehicles and their effects on the electricity supply industry. These scenarios 
TABLE III

SCENARIOS FOR THE DEPLOYMENT OF PH AND EV'S

\begin{tabular}{|c|c|c|}
\hline Scenario & Charging & Oil Prices \\
\hline 1 & Uncontrolled & Reference \\
\hline 2 & Uncontrolled & High \\
\hline 3 & Off-Peak & Reference \\
\hline 4 & Off-Peak & High \\
\hline
\end{tabular}

\section{RESULTS}

\section{A. Effects on the Availability Electricity}

The effects on the deployment rates of PH and EV's on the availability and security of supply of energy is represented by the results in Table IV. Scenarios 1 and 2 represent a significant growth in the observed rate of Unserved Energy within the NEM. Uncontrolled charging of PH and EV's with two different growth rates of deployment suggests that evening peak has significantly grown beyond the available capacity of peaking and intermediate electricity generation assets. Constraints on gas supply and high prices over this period have prevented the appropriate installation of this generation stock.

The controlled charging scenarios (3 and 4) represent a significantly lower rate of observation of unserved energy across the NEM. The off-peak charging profile of these scenarios places less strain on the growing peak in demand. The pervasiveness of higher energy consumption is growing peak energy demand across electricity supply industries across the developed world. Controlled charging represents an option for avoided peak growth and ensuring the security of supply of energy on the NEM.

TABLE IV

UNSERVED ENERGY FOR THE FORECASTED DEPLOYMENT OF EV'S (GWH)

\begin{tabular}{|l|c|c|c|c|}
\hline & Scenario 1 & Scenario 2 & Scenario 3 & Scenario 4 \\
\hline 2020 & 6.16 & 6.90 & 52.19 & 5.86 \\
\hline 2021 & 20.21 & 23.18 & 52.33 & 31.58 \\
\hline 2022 & 14.58 & 16.39 & 40.85 & 18.05 \\
\hline 2023 & 14.39 & 24.93 & 64.33 & 31.89 \\
\hline 2024 & 37.22 & 52.32 & 75.20 & 19.43 \\
\hline 2025 & 64.58 & 140.01 & 82.85 & 68.43 \\
\hline 2026 & 290.80 & 590.90 & 181.89 & 190.96 \\
\hline 2027 & 331.84 & 539.21 & 175.62 & 146.77 \\
\hline 2028 & 781.60 & 1133.37 & 300.43 & 291.02 \\
\hline 2029 & 1541.03 & 2240.83 & 505.18 & 570.15 \\
\hline 2030 & 3338.33 & 3472.52 & 657.07 & 660.26 \\
\hline
\end{tabular}

\section{B. Effects on Spot Prices}

The effects of $\mathrm{PH}$ and EV charging behaviour on the security of supply of energy is clearly reflected on spot market prices. The incidence of high and volatile prices in the NEM (for scenarios 1 and 2, see Table V), indicates a significant shortening of the reserve plant margin and the low availability of rapid start peaking power stations. While the incidence of VoLL (currently set at $\$ 12,500$ ) is ever present, the number of spikes is significantly lower due to off-peak charging (scenarios 3 and 4). Lower price
TABLE V

Average Spot Prices (\$/MWh ) FOR THE FORECASTED DEPLOYMENT OF EV'S

\begin{tabular}{|l|c|c|c|c|}
\hline & Scenario 1 & Scenario 2 & Scenario 3 & Scenario 4 \\
\hline 2020 & $\$ 40.89$ & $\$ 39.33$ & $\$ 75.46$ & $\$ 45.29$ \\
\hline 2021 & $\$ 56.88$ & $\$ 64.15$ & $\$ 82.30$ & $\$ 66.80$ \\
\hline 2022 & $\$ 55.86$ & $\$ 62.15$ & $\$ 88.86$ & $\$ 55.19$ \\
\hline 2023 & $\$ 62.53$ & $\$ 81.43$ & $\$ 105.78$ & $\$ 71.24$ \\
\hline 2024 & $\$ 110.14$ & $\$ 123.75$ & $\$ 114.57$ & $\$ 73.56$ \\
\hline 2025 & $\$ 152.92$ & $\$ 229.62$ & $\$ 147.86$ & $\$ 148.59$ \\
\hline 2026 & $\$ 443.07$ & $\$ 649.24$ & $\$ 287.67$ & $\$ 289.13$ \\
\hline 2027 & $\$ 490.47$ & $\$ 621.05$ & $\$ 272.43$ & $\$ 256.19$ \\
\hline 2028 & $\$ 851.36$ & $\$ 995.14$ & $\$ 438.18$ & $\$ 401.29$ \\
\hline 2029 & $\$ 1,415.53$ & $\$ 1,489.92$ & $\$ 617.47$ & $\$ 658.96$ \\
\hline 2030 & $\$ 3,613.25$ & $\$ 2,310.23$ & $\$ 833.76$ & $\$ 852.93$ \\
\hline
\end{tabular}

\section{Effects on Emissions}

The construction of off peak charging profiles (scenarios 3 and 4) has had an upward lift in the emissions intensity of electricity generated to serve vehicle battery charging (see Table VI. The average emissions intensity profile of base load coal is almost double that of CCGT plant [1]. Therefore shifting generation requirements to off peak base load assets represent a small increase relative to the total NEM emissions. Conversely Scenarios 1 and 2 impose a very small increase in relative emissions dude to the availability of CCGT plant.

TABLE VI

EMISSIONS FROM ELECTRICITY GENERATION ON THE NEM(MT-CO $\left.{ }_{2}\right)$

\begin{tabular}{|l|c|c|c|c|}
\hline & Scenario 1 & Scenario 2 & Scenario 3 & Scenario 4 \\
\hline 2020 & 228 & 218 & 229 & 218 \\
\hline 2021 & 234 & 225 & 235 & 225 \\
\hline 2022 & 239 & 231 & 240 & 232 \\
\hline 2023 & 247 & 240 & 248 & 240 \\
\hline 2024 & 254 & 248 & 256 & 248 \\
\hline 2025 & 260 & 254 & 263 & 255 \\
\hline 2026 & 269 & 266 & 271 & 266 \\
\hline 2027 & 269 & 266 & 271 & 266 \\
\hline 2028 & 275 & 271 & 276 & 271 \\
\hline 2029 & 279 & 274 & 279 & 275 \\
\hline 2030 & 282 & 278 & 284 & 280 \\
\hline
\end{tabular}




\section{CONCLUSION}

The results of our simulations indicate that wholesale prices during the off-peak period will increase slowly over time with controlled charging. While uncontrolled charging increases the incidence of extreme price events and a considerable number of hours with unserved energy within the network. This increase in spot prices will require further review by policy makers of regulated retail electricity tariffs. We expect the transfer of greenhouse gas emissions from petrol fueled personal transportation to electricity generation will assist Australia in achieving emissions reductions from the transport sector. Our results also indicate a variety of demand scenarios which will impact on spot prices throughout the NEM. Increases to spot price exposure for electricity retail firms could signal possible tariff price restructuring for retail consumers.

\section{REFERENCES}

[1] ACIL Tasman (2009), Fuel resource, new entry and generation costs in the NEM: Final Report 9. Report prepared by ACIL Tasman for the Interregional Planning Committee (IRPC).

[2] AEMO. Annual National Transmission Survey, 2009 http://www.aemo.com.au

[3] AEMO. Statement of Opportunities, 2009 http://www.aemo.com.au

[4] AEMO. Electricity Market Data, 2010 http://www.aemo.com.au

[5] CSIRO and ABARE, Modelling Energy Futures Forum Scenarios Using ESM, Canberra: CSIRO, December, (2006) http://www.csiro.au/files/files/pb63.pdf

[6] CSIRO, Fuel for Thought - The Future of Transport Fuels: Challenges and Opportunities, A Report by the Future Fuels Forum, Canberra: CSIRO, June, (2008) http://www.csiro.au/files/files/plm4.pdf

[7] EIA, Annual Energy Outlook 2010: With Projections to 2035, DOE/EIA0383(2010), Washington DC: U.S. Energy Information Administration, April, (2010)

[8] Energy Exemplar, Pty Ltd. P.O. Box 13, North Adelaide, SA 5006, Adelaide, Australia, http://www.energyexemplar.com

[9] EPRI, Comparing the Benefits and Impacts of Hybrid Electric Vehicle Options, EPRI, Palo Alto, CA, 2001. 111349

[10] EPRI, Environmental Assessment of Plug-in Hybrid Electric Vehicles, vol. 1, Nationwide Greenhouse Gas Emissions:, 10153525, EPRI: Palo Alto, CA, 2007

[11] Garnaut, R., Garnaut Climate Change Review Final Report, Port Melbourne: Cambridge University Press, (2008)

[12] Graham, P., Reedman, L. and Poldy, F., Modelling of the Future of Transport Fuels in Australia: A Report to the Future Fuels Forum, CSIRO Report No. ET/IR 1046, June, (2008) http://www.csiro.au/resources/FuelForThoughtModelling.html

[13] Graham, P. and Reedman, L., Peak oil and energy security: can alternative fuels and vehicles save us?, International Journal of Global Energy Issues, (2010), vol. 33 no. 1/2, 22-37.

[14] IEA, World Economic Outlook 2009, Paris: IEA, November, (2010)

[15] Parks, K., Denholm P., Markel, T., Costs and Emissions Associated with Plug-in Hybrid Electric Vehicle Charging in the Xcel Energy Colorado Service Territory, Technical Report NREL/TP-640-41410 May (2007), National Renewable Energy Laboratory, Midwest Research Institute, Battelle, CO, USA.

[16] Reedman, L. and Graham, P., Emissions Trading and the Convergence of Electricity and Transport Markets in Australia, in Evans, J. and Hunt, L. (eds), International Handbook on the Economics of Energy, Aldershot, UK: Edward Elgar, 685-704. (2009) 